Ciência Florestal, Santa Maria, v. 23, n. 1, p. 1-17, jan.-mar., 2013

ISSN 0103-9954

\title{
CONSERVAÇÃO DAS FLORESTAS DO PROJETO DE ASSENTAMENTO BENFICA, SUDESTE DAAMAZÔNIA
}

\author{
CONSERVATION OF THE FORESTS AT BENFICA SETTLEMENT PROJECT IN SOUTHEASTERN \\ AMAZON
}

Roberta de Fátima Rodrigues Coelho ${ }^{1}$ Izildinha de Souza Miranda ${ }^{2}$ Danielle Mitja ${ }^{3}$

\section{RESUMO}

Este trabalho teve como objetivo descrever e analisar a composição florística e a estrutura de remanescentes florestais do Projeto de Assentamento Benfica, no município de Itupiranga, Pará. Foram selecionadas 11 áreas de florestas localizadas em diferentes propriedades e com diferentes níveis de perturbação. No interior de cada área foi inventariada a vegetação em um transecto de $500 \mathrm{~m}^{2}$, considerando três estratos: superior, médio e inferior. A vegetação foi classificada em cinco formas de vida: árvores, arbustos, lianas, palmeiras e herbáceas; e dois grupos funcionais: pioneiras e florestais. Foram encontradas 286 espécies, 173 gêneros e 68 famílias. A maioria das espécies lenhosas (51\%) ocorreu em apenas um estrato. A riqueza de espécies correspondeu a $70 \%$ da riqueza estimada por Jack-nife de primeira ordem $\left(\mathrm{J}^{1}=418,45\right)$. A porcentagem de espécies raras foi alta; 138 espécies ocorreram em apenas uma área. Em todos os estratos, as árvores apresentaram maior densidade, exceto no estrato inferior, onde as herbáceas foram mais densas, seguida das árvores. As espécies florestais predominaram em todos os estratos. Houve diferença significativa entre as florestais e pioneiras em todas as formas de vida, em todos os estratos. A análise de componentes principais mostrou heterogeneidade entre os transectos estudados, formando três grupos que parecem estar relacionados com os diferentes níveis de perturbações.

Palavras-chave: formas de vida; grupos funcionais; riqueza.

\begin{abstract}
In this study we analyze the floristic and structure of forest remnants in Benfica Settlement Project located in the municipality of Itupiranga, in southeastern Amazon. Eleven areas of forests located in different properties and different levels of disturbance were selected. Within each area, plants in one plot of $500 \mathrm{~m}^{2}$, were surveyed considering three strata: upper, middle and inferior. The species were classified in five life forms: tree, scrub, liana, palm and herb; and two functional groups, the pioneer and forest species. Two houndred and eighty-six (286) species, 173 genera and 68 families were found. Most woody species (51 \%) occurred in only one stratum. The richness of species was $70 \%$ of estimated richness by Jack-nife of first order. The percentage of rare species was high, 138 species occurred in only one area. In all strata, the trees had higher density, except in inferior stratum, where the grasses were denser. The forest species, in all strata, were dominant. There was significant difference between the forest and pioneer in all forms of life, in all strata. The principal components analysis showed heterogeneity among the studied plots, forming three groups which appear to be related to the different disturbance levels.
\end{abstract}

Keywords: life forms; functional groups; richness.

1. Engenheira Florestal, Dra., Professora, Instituto Federal de Educação, Ciência e Tecnologia do Pará, Campus Castanhal, Rodovia BR-316, Km 62 s/n, CEP 68740-970, Castanhal (PA). roberta.fatimacoelho@gmail.com

2. Bióloga, Dra., Professora Associada do Instituto Sócio Ambiental e de Recursos Hídricos, Universidade Federal Rural da Amazônia, Caixa Postal 817, CEP 66077-000, Belém (PA). Izildinha.miranda@ufra.edu.br

3. Bióloga, Dra., Institut de Recherche pour le Développement, Instituto de pesquisa para o desenvolvimento, UMR 228- ESPACE-DEV, 500, Rue Jean François Breton, 34093 Montpellier, Cedex 05, França. danielle.mitja@ird.fr Recebido para publicação em 22/06/2009 e aceito em 25/10/2011 


\section{INTRODUÇÃO}

$\mathrm{Na}$ Amazônia brasileira o processo de fragmentação das florestas ocorre especialmente devido à supressão das florestas para instalação de pastagens (MUCHAGATA e BROWN, 2003) e grande parte das áreas desmatadas está ligada aos Projetos rodoviários planejados (SOARES-FILHO et al., 2004). Embora, em termos regionais, as grandes fazendas sejam responsáveis pela maior parte das áreas desmatadas, em algumas regiões os pequenos produtores são os mais importantes. Por exemplo, ao longo da rodovia Transamazônica, no Estado do Pará, os Projetos de Assentamento (PA) caracterizados pela agricultura familiar têm sido apontados como os principais causadores do desmatamento (FEARNSIDE, 2006).

Embora tenha havido um grande aumento no número de projetos de assentamentos na Amazônia, as elevadas taxas de evasão (no Estado do Pará a evasão chegam a $60 \%$ ) e de desflorestamento comprovam a falta de sustentabilidade econômica e ambiental desses assentamentos (PASQUIS et al., 2005).

A fragmentação das florestas tropicais provoca mudanças na estrutura e dinâmica das comunidades, na diversidade das espécies e nas interações bióticas existentes, como as causadas pela infestação de cipós, pelo aumento de espécies invasoras, pela interrupção do fluxo gênico e pelo aumento da mortalidade de animais e plantas. A fragmentação também provoca aumento da temperatura, radiação solar e evapotranspiração, redução da umidade relativa e da retenção da precipitação, aumento do risco de erosão, assoreamento de cursos d'água e diminuição de resistência à ação do vento (BIERREGAARD et al., 2001).

Os fragmentos florestais remanescentes em áreas alteradas podem servir como áreas tampões diante de uma variação de microclima, ajudam a reduzir a perda de água e erosão, além de aumentar a biodiversidade nas áreas de plantações ou pastagens influenciando especialmente em nível de paisagens (PIMENTEL et al., 1992). As espécies existentes nesses fragmentos florestais também influenciam fortemente os padrões de regeneração florestal nas áreas agrícolas (NEPSTAD et al., 1996; DUNCAN, 2006; VIEIRA e PROCTOR, 2007).

Os processos ecológicos dos fragmentos florestais nas áreas agrícolas, além de serem influenciados pela fragmentação, são também influenciados pela dinâmica da vegetação na matriz agrícola (DAUBER et al., 2003; KÖHLER et al., 2003).

Na Amazônia brasileira, as principais ações para conservação da biodiversidade têm sido voltadas principalmente para as unidades de conservação (BRANDON et al., 2005). Embora essas ações estejam sendo bem sucedidas (MITTERMEIER et al., 2005), alguns autores apontam para a necessidade de conservação das florestas em áreas agrícolas, estabelecendo um novo paradigma da conservação (PIMENTEL et al., 1992; FOX et al., 2000; TREWAVAS, 2001; VANDERMEER e CARVAJAL, 2001; VANDERMEER e PERFECTO, 2005, 2007).

Um manejo adequado nas áreas agrícolas pode ajudar na conservação dos fragmentos florestais remanescentes, assim como em toda a matriz agrícola. Algumas sugestões nesse sentido têm sido apontadas (PIMENTEL et al., 1992; LAURANCE e GASCON, 1997; CULLEN et al., 2005), por exemplo, a conservação de habitats remanescentes dentro da matriz agrícola (HARVEY et al., 2007), pois estes oferecem recursos para as espécies nativas, mantém intactas as comunidades ecológicas, servem como fonte genética para a recolonização da matriz agrícola e protege contra os eventos do tempo e as mudanças climáticas (BENGTSSON et al., 2003; TABARELLI e GASCON, 2005).

Este trabalho tem como objetivo descrever e analisar a composição florística e a estrutura de remanescentes florestais em uma área agrícola, no sudeste do Estado do Pará, visando verificar a situação de conservação dessas florestas.

\section{MATERIAL E MÉTODOS}

\section{Área de estudo}

O trabalho foi realizado no Projeto de Assentamento Benfica (PA-Benfica) que está inserido na mesorregião Sudeste Paraense, microrregião Tucurí (MRH-016), Município de Itupiranga. O PA-Benfica possui uma área de $10.026,00$ hectares e localiza-se entre as coordenadas $05^{\circ} 12^{\prime} 20^{\prime \prime}$ e $05^{\circ} 20^{\prime} 40^{\prime \prime}$ de latitude Sul e $49^{\circ} 56^{\prime} 40^{\prime \prime}$ e 49 $48^{\prime} 00^{\prime \prime}$ 'de longitude Oeste, no km-15 da vicinal do Rio da Esquerda, a 70 quilômetros da BR230 (Transamazônica), distante 85 quilômetros da sede municipal e 120 quilômetros da cidade de Marabá (SAMPAIO, 2008).

O clima é caracterizado por uma temperatura 
média anual de $26{ }^{\circ} \mathrm{C}$ e $2000 \mathrm{~mm}$ de pluviosidade anual, com uma estação seca bem definida entre maio e setembro (REYNAL et al., 1995). Os solos mudam de acordo com a topografia. No alto das colinas os latossolos predominam nos declives os cambissolos são mais comuns, e nos baixios ocorrem os solos hidromórficos. As maiores limitações dos solos estão ligadas à fertilidade química. Os solos são ácidos, com pouca ou nenhuma reserva mineral (SOMBROEK, 2000). Os latossolos são bem estruturados, profundos ou muito profundos e bem drenados. Pode existir risco de déficit hídrico, principalmente nos cambissolos. Concreções lateríticas podem existir próximo à superfície, o que leva a dificuldade para o desenvolvimento das raízes (REYNAL et al., 1995).

A vegetação típica da região é uma floresta tropical úmida de terra firme (RADAMBRASIL, 1974), caracterizada pela presença de lianas e palmeiras arborescentes. A área de estudo está localizada na região conhecida como polígono dos castanhais, conhecida pela alta densidade de Bertholletia excelsa H.B.K.

Os sítios de estudo pertencem a uma comunidade de pequenos produtores que foram assentados em 1994 sobre uma área de 6.000 ha, que anteriormente pertencia a duas grandes fazendas. A área média de cada proprietário é de 50 ha. A paisagem de Benfica é caracterizada por um mosaico de diferentes coberturas, como florestas de terra firme, florestas ripárias, florestas secundárias, brejos e roças.

Os fragmentos florestais apresentam cerca de três hectares, em média, e apresentam várias formas. Em 2005, a área florestal correspondia $25 \%$ da cobertura vegetal do PA-Benfica (SAMPAIO, 2008). Os fragmentos florestais são utilizados para a subsistência dos agricultores (caça, coleta de frutos, sementes e madeira) e rodeados principalmente por pastagens e florestas secundárias.

\section{Coleta de dados}

Foram selecionadas 11 áreas de fragmentos florestais localizadas em diferentes propriedades e com diferentes níveis de perturbação. No interior de cada área foi implantado um transecto de $10 \times 50 \mathrm{~m}$, localizado no mínimo a $50 \mathrm{~m}$ da borda. No transecto foram inventariados todos os indivíduos com diâmetro a altura do peito (DAP) $\geq 10 \mathrm{~cm}$ (Estrato superior); dentro desse transecto, foi alocado um subtransecto de $5 \times 50 \mathrm{~m}$, onde foram inventariados todos os indivíduos com DAP $<10 \mathrm{~cm}$ e altura $\geq$ a 2,0 m (Estrato médio); dentro desse subtransecto foi alocado um segundo subtransecto de $1 \times 50 \mathrm{~m}$, onde foram inventariados os indivíduos com altura $<2,0 \mathrm{~m}$ (Estrato inferior).

Foi medido o diâmetro a altura de $1,30 \mathrm{~m}$ do solo (DAP) no estrato superior e médio. Em todos os estratos foram coletados ramos férteis ou estéreis para a identificação por comparação ao acervo do Herbário João Murça Pires, do Museu Paraense Emílio Goeldi; as plantas superiores foram classificadas de acordo com o sistema do Angiosperm Phylogeny Group II (APG II, 2003) e as samambaias foram classificadas de acordo com Kramer e Green (1990).

As espécies foram classificadas em cinco formas de vida: árvores, arbustos, lianas, palmeiras e herbáceas. Nesse último grupo foram reunidas as herbáceas sensu stricto, herbáceas escandentes, epífitas e hemiepífitas. Essas formas de vida foram classificadas em dois diferentes grupos funcionais, conforme Mitja et al. (2008): espécies pioneiras (1) são aquelas bem conhecidas na literatura e normalmente encontradas em ambientes abertos, em clareiras dentro da floresta, normalmente caracterizadas pela capacidade de formar banco de sementes viáveis por longo tempo; e, espécies florestais (2) são aquelas normalmente encontradas na floresta em ambientes não perturbados (no piso florestal, no sub-bosque e no dossel).

\section{Análise de dados}

Para análise florística e estrutural foram considerados: densidade de indivíduos (ind. $/ \mathrm{m}^{2}$ ), riqueza de espécies $(\mathrm{S})$, diversidade de Shannon (H'), equibilidade (E) usando o índice de Pielou, segundo Magurran (1998). A riqueza de espécies foi comparada à riqueza estimada por meio do índice não paramétrico de Jack-nife de primeira ordem $\left(\mathrm{J}^{1}\right)$ $=\mathrm{SO}+\mathrm{r} 1(\mathrm{n}-1) / \mathrm{n}$ e o de Jack-nife de segunda ordem $\left(\mathrm{J}^{2}\right)=\mathrm{SO}+\{[\mathrm{r} 1(2 \mathrm{n}-3)] / \mathrm{n}-[\mathrm{r} 2(\mathrm{n}-2)] /[\mathrm{n}(\mathrm{n}-1)]\}$, onde: $\mathrm{SO}=$ número total de espécies que ocorreram em $\mathrm{n}$ áreas; $\mathrm{r} 1=$ número de espécies que ocorreu em apenas uma parcela; r2 número de espécies que ocorreram em duas parcelas (PALMER, 1990; 1991).

As médias do número de espécies pioneiras e florestais por tipo biológico foram comparadas por análise de variância de um critério e quando diferentes espécies foram testadas com o teste de Bonferroni (B) devido ao pequeno número de amostras por estágio, conforme recomendado por Zar (1996). 
A composição florística foi analisada por meio de uma análise de componente principal (PCA) realizada pela matriz de variância dos dados de abundância de 110 espécies que ocorreram em pelo menos três das onze áreas estudadas. A abundância foi transformada através do índice de favorabilidade sociológica, recomendado por Mccune (1994) para comunidades heterogêneas, que apresentam uma matriz de dados com um número grande de zeros.

Foi utilizado o programa (IndVAL) para a análise das espécies indicadoras dos grupos mostrados pela PCA. Espécies indicadoras são definidas como as mais características de cada grupo, encontradas principalmente em um único grupo da tipologia e presente na maioria dos locais que pertencem àquele grupo (DUFRENE e LEGENDRE, 1997). O método assume que dois ou mais grupos a priori estabelecidos existem e que as abundâncias das espécies foram tomadas em cada uma das áreas. O IndVal considera as densidades e frequências das espécies dentro dos grupos préestabelecidos, alcança valores entre 0 e 100 e o IndVal $_{\text {max }}$ é o maior valor alcançado pela espécie nas várias repartições. $O$ teste de Monte Carlo, com 500 repetições, foi utilizado para determinar os valores de IndVal ${ }_{\text {max }}$ significantes.

\section{RESULTADOS}

Nos transectos estudados foram encontradas 286 espécies, 173 gêneros e 67 famílias (Tabela 1). As famílias com maior densidade de espécies foram Fabaceae (47 espécies), Sapindaceae (14), Annonaceae e Bignoniaceae (13 espécies cada), Moraceae (12) e Arecaceae, Malvaceae e Meliaceae (10 espécies cada). Nos onze transectos estudados, o gênero Inga foi predominante com 14 espécies, seguido dos gêneros Guarea (6 espécies), Bauhinia, Cordia, Memora (5 espécies cada), Adiantum, Calathea, Theobroma, Protium, Pouteria, Ocotea, Talisia (4 espécies cada), Apeiba, Arrabidea, Cecropia, Duguetia, Piper, Eugenia, Swartzia, Aspidosperma (3 espécies cada).

Dentre as 248 espécies lenhosas identificadas, a maioria das espécies (51\%) ocorreu em apenas um estrato ( 25 espécies ocorreram apenas no estrato superior, 52 no estrato médio e 51 no estrato inferior), 39 espécies (15\%) ocorreram nos três estratos e $32 \%$ ( 81 espécies) ocorreram em dois estratos (Tabela 1).

TABELA 1: Famílias e espécies encontradas nos 11 fragmentos florestais estudados no PA-Benfica, Itupiranga, Pará. Formas de vida e grupo funcional: HF: herbácea florestal, HP: herbácea pioneira, AF: árvore florestal, AP: árvore pioneira, ArbF: arbusto florestal, ArbP: arbusto pioneira, PalmF: Palmeira florestal, LF: liana florestal; LP: liana pioneira.

TABLE 1: Families and species in the 11 forest fragments studied in PA-Benfica, Itupiranga, Pará state. Life forms and functional group: HF: forest herbs, HP: pioneer herbs, AF: forest tree, AP: pioneer tree, ArbF: forest shrub, ArbP: pioneer shrub, PalmF: forests Palm, LF: forest liana, LP: pioneer liana.

\begin{tabular}{|c|c|c|c|c|}
\hline Família/Espécie & $\begin{array}{l}\text { Forma de vida e } \\
\text { grupo funcional }\end{array}$ & Estrato superior & Estrato médio & $\begin{array}{l}\text { Estrato } \\
\text { inferior }\end{array}$ \\
\hline 1. ACHARIACEAE & & & & \\
\hline Carpotroche sp. & $\mathrm{AF}$ & & $\mathrm{X}$ & $\mathrm{X}$ \\
\hline \multicolumn{5}{|l|}{ 2. ANACARDIACEAE } \\
\hline Astronium gracile Engl. & $\mathrm{AF}$ & $\mathrm{X}$ & $\mathrm{X}$ & $\mathrm{X}$ \\
\hline Astronium lecointei Ducke & $\mathrm{AF}$ & $\mathrm{X}$ & & $\mathrm{X}$ \\
\hline Thyrsodium paraense Huber & $\mathrm{AF}$ & $\mathrm{X}$ & & \\
\hline \multicolumn{5}{|l|}{ 3. ANNONACEAE } \\
\hline Anaxagorea dolichocarpa Sprague \& Sandwith & $\mathrm{AF}$ & $\mathrm{X}$ & $\mathrm{X}$ & $\mathrm{X}$ \\
\hline Annona montana Macfad. & AP & & $\mathrm{X}$ & $\mathrm{X}$ \\
\hline $\begin{array}{l}\text { Cymbopetalum brasiliense (Vell.) Benth. Ex } \\
\text { Baill. }\end{array}$ & ArbF & & $\mathrm{X}$ & \\
\hline Duguetia echinophora R.E. Fr. & AP & $\mathrm{X}$ & $\mathrm{X}$ & $\mathrm{X}$ \\
\hline Duguetia flagellaris Huber & $\mathrm{AF}$ & & $\mathrm{X}$ & $\mathrm{X}$ \\
\hline Duguetia surinamensis R.E. Fr. & $\mathrm{AF}$ & & $\mathrm{X}$ & \\
\hline Ephedranthus pisocarpus R. E. Fr. & ArbP & & $\mathrm{X}$ & \\
\hline Guatteria poeppigiana Mart. & $\mathrm{AF}$ & & $\mathrm{X}$ & \\
\hline
\end{tabular}


TABELA 1: Continuação ...

TABLE 1: Continued ...

\begin{tabular}{|c|c|c|c|c|}
\hline Família/Espécie & $\begin{array}{l}\text { Forma de vida e } \\
\text { grupo funcional }\end{array}$ & Estrato superior & Estrato médio & $\begin{array}{l}\text { Estrato } \\
\text { inferior }\end{array}$ \\
\hline Rollinia exsucca (DC. ex Dunal) A. DC. & $\mathrm{AF}$ & $\mathrm{X}$ & & $\mathrm{X}$ \\
\hline Unonopsis cf. guatteroides (A.DC.) R.E. Fr. & $\mathrm{AF}$ & $\mathrm{X}$ & $\mathrm{X}$ & \\
\hline Xylopia amazonica R. E. Fr. & $\mathrm{AF}$ & & & $\mathrm{X}$ \\
\hline Xylopia cayennensis Maas & $\mathrm{AF}$ & & $\mathrm{X}$ & $\mathrm{X}$ \\
\hline Xylopia nitida Dunal & $\mathrm{AF}$ & $\mathrm{X}$ & & $\mathrm{X}$ \\
\hline \multicolumn{5}{|l|}{ 4. APOCYNACEAE } \\
\hline Aspidosperma desmathum Benth. & $\mathrm{AF}$ & $\mathrm{X}$ & & \\
\hline Aspidosperma excelsum Benth. & $\mathrm{AF}$ & & $\mathrm{X}$ & $\mathrm{X}$ \\
\hline Aspidosperma nitidum Benth. ex Müll. Arg. & $\mathrm{AF}$ & & $\mathrm{X}$ & \\
\hline Geissospermum vellosii Allemão & $\mathrm{AF}$ & $\mathrm{X}$ & $\mathrm{X}$ & $\mathrm{X}$ \\
\hline \multicolumn{5}{|l|}{ 5. ARACEAE } \\
\hline \multicolumn{4}{|l|}{ 6. ARALIACEAE } & $\mathrm{X}$ \\
\hline $\begin{array}{l}\text { Schefflera morototoni (Aubl.) Maguire, } \\
\text { Steyerm. \& Frodin }\end{array}$ & $\mathrm{AF}$ & & $\mathrm{X}$ & \\
\hline \multicolumn{5}{|l|}{ 7. ARECACEAE } \\
\hline Astroçaryum gynacanthum Mart. & PalmF & & $\mathrm{X}$ & $\mathrm{X}$ \\
\hline Attalea maripa (Aubl.) Mart. & PalmF & & & $\mathrm{X}$ \\
\hline Attalea speciosa Mart. ex Spreng. & PalmF & $\mathrm{X}$ & $\mathrm{X}$ & $\mathrm{X}$ \\
\hline Bactris maraja Mart. & PalmF & & $\mathrm{X}$ & $\mathrm{X}$ \\
\hline Euterpe oleracea Mart. & PalmF & & & $\mathrm{X}$ \\
\hline Geonoma baculifera (Poit.) Kunth & PalmF & & & $\mathrm{X}$ \\
\hline Geonoma maxima (Poit.) Kunth & PalmF & & $\mathrm{X}$ & \\
\hline Iriartea exorrhiza Mart. & PalmF & $\mathrm{X}$ & & $\mathrm{X}$ \\
\hline Oenocarpus bacaba Mart. & PalmF & & & $\mathrm{X}$ \\
\hline $\begin{array}{l}\text { Oenocarpus distichus Mart. } \\
\text { 8. BIGNONIACEAE }\end{array}$ & PalmF & $\mathrm{X}$ & $\mathrm{X}$ & $\mathrm{X}$ \\
\hline Anemopaegma sp. & $\mathrm{HF}$ & & & $\mathrm{X}$ \\
\hline Arrabidaea cinnamomea (A. DC.) Sandwith & $\mathrm{HF}$ & & & $\mathrm{X}$ \\
\hline Arrabidaea florida A. DC. & HP & & & $\mathrm{X}$ \\
\hline Arrabidaea sp. & $\mathrm{HF}$ & & & $\mathrm{X}$ \\
\hline Jacaranda copaia (Aubl.) D.Don & $\mathrm{AF}$ & $\mathrm{X}$ & $\mathrm{X}$ & $\mathrm{X}$ \\
\hline Memora allamandiflora Bureau ex K. Schum. & $\mathrm{LF}$ & & $\mathrm{X}$ & $\mathrm{X}$ \\
\hline Memora contracta A.H. Gentry ex Hauk & LF & & & $\mathrm{X}$ \\
\hline Memora flavida (DC.) Bureau ex K. Schum. & $\mathrm{LF}$ & & $\mathrm{X}$ & $\mathrm{X}$ \\
\hline Memora magnifica (Mart. ex DC.) Bureau & $\mathrm{LF}$ & & & $\mathrm{X}$ \\
\hline Memoracf. flaviflora (Miq.) Pulle & $\mathrm{LF}$ & & & $\mathrm{X}$ \\
\hline Pachyptera kerere (Aubl.) Sandwith & $\mathrm{LF}$ & & & $\mathrm{X}$ \\
\hline Pleonotoma cf. dendrotricha Sandwith & $\mathrm{LF}$ & & & $\mathrm{X}$ \\
\hline Pleonotoma jasminifolia (Kunth) Miers & $\mathrm{LF}$ & & & $\mathrm{X}$ \\
\hline \multicolumn{5}{|l|}{ 9. BORAGINACEAE } \\
\hline Cordia lomatoloba I.M.Johnst. & AP & & $\mathrm{X}$ & \\
\hline Cordia nodosa Lam. & $\mathrm{AF}$ & & $\mathrm{X}$ & \\
\hline Cordia scabrida Mart. & $\mathrm{AF}$ & $\mathrm{X}$ & $\mathrm{X}$ & \\
\hline Cordia scabrifolia A. DC. & AP & $\mathrm{X}$ & $\mathrm{X}$ & \\
\hline \multicolumn{5}{|l|}{ 10. BURSERACEAE } \\
\hline $\begin{array}{l}\text { Crepidospermum goudotianum (Tul.) Triana \& } \\
\text { Planch. }\end{array}$ & $\mathrm{AF}$ & & $\mathrm{X}$ & $\mathrm{X}$ \\
\hline Protium apiculatum Swart & $\mathrm{AF}$ & $\mathrm{X}$ & $\mathrm{X}$ & $\mathrm{X}$ \\
\hline
\end{tabular}


TABELA 1: Continuação ...

TABLE 1: Continued ...

\begin{tabular}{|c|c|c|c|c|}
\hline Família/Espécie & $\begin{array}{l}\text { Forma de vida e } \\
\text { grupo funcional }\end{array}$ & Estrato superior & Estrato médio & $\begin{array}{l}\text { Estrato } \\
\text { inferior }\end{array}$ \\
\hline Protium aracouchini (Aubl.) Marchand & $\mathrm{AF}$ & $\mathrm{X}$ & & \\
\hline Protium heptaphyllum (Aubl.) Marchand. & $\mathrm{AF}$ & & $\mathrm{X}$ & \\
\hline Protium insigne (Triana \& Planch.) Engl. & $\mathrm{AF}$ & & $\mathrm{X}$ & \\
\hline Tetragastris altissima (Aubl.) Swart & $\mathrm{AF}$ & $\mathrm{X}$ & $\mathrm{X}$ & $\mathrm{X}$ \\
\hline Tetragastris panamensis (Engl.) Kuntze & $\mathrm{AF}$ & & $\mathrm{X}$ & \\
\hline $\begin{array}{l}\text { Trattinickia rhoifolia Willd. } \\
\text { 11. CAPPARACEAE }\end{array}$ & $\mathrm{AF}$ & & $\mathrm{X}$ & \\
\hline $\begin{array}{l}\text { Capparis amazonica } \text { H. H. Iltis } \\
\text { 12. CARICACEAE }\end{array}$ & $\mathrm{AF}$ & & $\mathrm{X}$ & $\mathrm{X}$ \\
\hline $\begin{array}{l}\text { Jacaratia spinosa (Aubl.) A.DC. } \\
\text { 13. CHRYSOBALANACEAE }\end{array}$ & $\mathrm{AF}$ & $\mathrm{X}$ & $\mathrm{X}$ & \\
\hline $\begin{array}{l}\text { Conepia sp. } \\
\text { 14. CLUSIACEAE }\end{array}$ & $\mathrm{AF}$ & $\mathrm{X}$ & $\mathrm{X}$ & $\mathrm{X}$ \\
\hline Rheedia gardneriana Planch. \& Triana & AP & $\mathrm{X}$ & $\mathrm{X}$ & \\
\hline $\begin{array}{l}\text { Symphonia globulifera L. f. } \\
\text { 15. COMBRETACEAE }\end{array}$ & $\mathrm{AF}$ & $\mathrm{X}$ & $\mathrm{X}$ & $\mathrm{X}$ \\
\hline $\begin{array}{l}\text { Combretum rotundifolium Rich. } \\
\text { 16. COMMELIACEAE }\end{array}$ & LP & & $\mathrm{X}$ & \\
\hline Commelina sp. & HP & & & $\mathrm{X}$ \\
\hline $\begin{array}{l}\text { 17. CONNARACEAE } \\
\text { Connarus erianthus Benth. ex Baker } \\
\text { 18. CONVOLVULACEAE }\end{array}$ & $\mathrm{HF}$ & & $\mathrm{X}$ & \\
\hline $\begin{array}{c}\text { Bonamia grandiflora (A. Gray) Hallier f. } \\
\text { 19. COSTACEAE }\end{array}$ & $\mathrm{HP}$ & & $\mathrm{X}$ & \\
\hline $\begin{array}{l}\text { Costus arabicus L. } \\
\text { 20. CYPERACEAE }\end{array}$ & $\mathrm{HF}$ & & & $\mathrm{X}$ \\
\hline Scleria pterota C. Presl ex C.B. Clarke & $\mathrm{HF}$ & & & $\mathrm{X}$ \\
\hline $\begin{array}{l}\text { Scleria secans (L.) Urb } \\
\text { 21. DILLENIACEAE }\end{array}$ & HP & & & $\mathrm{X}$ \\
\hline $\begin{array}{l}\text { Doliocarpus dentatus (Aubl.) Standl. } \\
\text { 22. DRYOPTERIDACEAE }\end{array}$ & LF & & & $\mathrm{X}$ \\
\hline Dryopteris sp. & HP & & & $\mathrm{X}$ \\
\hline $\begin{array}{l}\text { Lomagramma guianensis (Aubl.) Ching } \\
\text { 23. EUPHORBIACEAE }\end{array}$ & $\mathrm{HF}$ & & & $\mathrm{X}$ \\
\hline Aparisthmium cordatum Baill. & $\mathrm{AF}$ & & $\mathrm{X}$ & $\mathrm{X}$ \\
\hline Dodecastigma amazonicum Ducke & ArbF & & $\mathrm{X}$ & \\
\hline $\begin{array}{l}\text { Dodecastigma integrifolium (Lanj.) Lanj.\& } \\
\text { Sandwith }\end{array}$ & ArbF & $\mathrm{X}$ & $\mathrm{X}$ & $\mathrm{X}$ \\
\hline Glycydendron amazonicum Ducke & $\mathrm{AF}$ & $\mathrm{X}$ & & \\
\hline $\begin{array}{l}\text { Manihot quinquepartita Huber ex Rogers \& } \\
\text { Appan }\end{array}$ & ArbF & & $\mathrm{X}$ & \\
\hline Pausandra trianae (Mull. Arg.) Baill. & $\mathrm{AF}$ & & & $\mathrm{X}$ \\
\hline Sagotia racemosa (Baill.) Müll.Arg. & $\mathrm{AF}$ & & $\mathrm{X}$ & $\mathrm{X}$ \\
\hline Sapium lanceolatum (Müll.Arg.) Huber & $\mathrm{AF}$ & $\mathrm{X}$ & & \\
\hline $\begin{array}{l}\text { Sapium marmieri Huber } \\
\text { 24. FABACEAE }\end{array}$ & $\mathrm{AF}$ & $\mathrm{X}$ & $\mathrm{X}$ & $\mathrm{X}$ \\
\hline Acacia multipinnata Ducke & $\mathrm{HF}$ & & $\mathrm{X}$ & $\mathrm{X}$ \\
\hline Alexa grandiflora Ducke & $\mathrm{AF}$ & $\mathrm{X}$ & $\mathrm{X}$ & \\
\hline Bauhinia acreana Harms & ArbF & $\mathrm{X}$ & $\mathrm{X}$ & \\
\hline Bauhinia guianensis Aubl. & ArbF & & & $\mathrm{X}$ \\
\hline Bauhinia longipedicellata Ducke & ArbF & $\mathrm{X}$ & & \\
\hline Bauhinia sp.1 & ArbF & & & $\mathrm{X}$ \\
\hline
\end{tabular}


TABELA 1: Continuação ...

TABLE 1: Continued ...

\begin{tabular}{|c|c|c|c|c|}
\hline Família/Espécie & $\begin{array}{l}\text { Forma de vida e } \\
\text { grupo funcional }\end{array}$ & Estrato superior & Estrato médio & $\begin{array}{l}\text { Estrato } \\
\text { inferior }\end{array}$ \\
\hline Bauhinia sp.2 & ArbF & & & $\mathrm{X}$ \\
\hline Cenostigma tocantinum Ducke & $\mathrm{AF}$ & $\mathrm{X}$ & & $\mathrm{X}$ \\
\hline Derris utilis (A.C.Smith) Ducke & HP & & & $\mathrm{X}$ \\
\hline Dialium guianense (Aubl.) Sandwith & $\mathrm{AF}$ & $\mathrm{X}$ & $\mathrm{X}$ & $\mathrm{X}$ \\
\hline Dioclea malacocarpa Ducke & $\mathrm{LF}$ & & & $\mathrm{X}$ \\
\hline Dioclea scleroçarpa Ducke & LF & & $\mathrm{X}$ & \\
\hline Enterolobium schomburgkii (Benth.) Benth. & $\mathrm{AF}$ & $\mathrm{X}$ & & $\mathrm{X}$ \\
\hline Hymenolobium flavum Kleinhoonte & $\mathrm{AF}$ & $\mathrm{X}$ & & \\
\hline Inga alba Willd. & $\mathrm{AF}$ & $\mathrm{X}$ & $\mathrm{X}$ & $\mathrm{X}$ \\
\hline Inga capitata Desv. & $\mathrm{AF}$ & $\mathrm{X}$ & $\mathrm{X}$ & $\mathrm{X}$ \\
\hline Inga chartacea Poepp. & $\mathrm{AF}$ & & $\mathrm{X}$ & \\
\hline Inga edulis Mart. & $\mathrm{AF}$ & $\mathrm{X}$ & $\mathrm{X}$ & $\mathrm{X}$ \\
\hline Inga flagelliformis (Vell.) Mart. & $\mathrm{AF}$ & $\mathrm{X}$ & & \\
\hline Inga laurina (Sw.) Willd. & $\mathrm{AF}$ & & $\mathrm{X}$ & \\
\hline Inga marginata Willd. & $\mathrm{AF}$ & $\mathrm{X}$ & & \\
\hline Inga rubiginosa (Rich.) DC. & $\mathrm{AF}$ & & & $\mathrm{X}$ \\
\hline Inga sp. & $\mathrm{AP}$ & & $\mathrm{X}$ & \\
\hline Inga stipularis DC. & $\mathrm{AF}$ & & $\mathrm{X}$ & $\mathrm{X}$ \\
\hline Inga thibaudiana DC. & AP & $\mathrm{X}$ & $\mathrm{X}$ & $\mathrm{X}$ \\
\hline Inga umbellifera (Vahl) Steud. & $\mathrm{AF}$ & & $\mathrm{X}$ & \\
\hline Inga umbratica Poepp. \& Endl. & AP & $\mathrm{X}$ & & \\
\hline Inga villosa $\mathrm{M}$. Martens \& Galeotti & $\mathrm{AF}$ & & & $\mathrm{X}$ \\
\hline Machaerium ferox (Benth.) Ducke & LF & & & $\mathrm{X}$ \\
\hline Machaerium madeirense Pittier & $\mathrm{LF}$ & & $\mathrm{X}$ & $\mathrm{X}$ \\
\hline Macherium sp. & HP & & $\mathrm{X}$ & \\
\hline Newtonia psilostachya Dc. Brenan & $\mathrm{AF}$ & & $\mathrm{X}$ & \\
\hline Ormosia coccinea Aubl. Jacks. & $\mathrm{AF}$ & $\mathrm{X}$ & & \\
\hline Poecilanthe effusa (Huber) Ducke & $\mathrm{AF}$ & $\mathrm{X}$ & $\mathrm{X}$ & $\mathrm{X}$ \\
\hline $\begin{array}{l}\text { Pseudopiptadenia suaveolens (Miq.) J.W. } \\
\text { Grimes }\end{array}$ & $\mathrm{AF}$ & $\mathrm{X}$ & & \\
\hline Pterocarpus rohrii Vahl & $\mathrm{AF}$ & & $\mathrm{X}$ & \\
\hline Pterocarpus santalinoides L'Hér. ex DC. & $\mathrm{AF}$ & $\mathrm{X}$ & & \\
\hline Sclerolobium paraense Huber & $\mathrm{AF}$ & $\mathrm{X}$ & $\mathrm{X}$ & \\
\hline Stryphnodendron cf. foreroi E.M.O. Martins & $\mathrm{AF}$ & & $\mathrm{X}$ & \\
\hline Stryphnodendron purpureum Ducke & $\mathrm{AF}$ & & $\mathrm{X}$ & \\
\hline Swartzia arborescens (Aubl.) Pitter & $\mathrm{AF}$ & & $\mathrm{X}$ & \\
\hline Swartzia argentea Spruce & $\mathrm{AF}$ & & $\mathrm{X}$ & \\
\hline Swartzia flaemingii Raddi & $\mathrm{AF}$ & & $\mathrm{X}$ & $\mathrm{X}$ \\
\hline Tachigalia myrmecophyla (Ducke) Ducke & $\mathrm{AP}$ & & $\mathrm{X}$ & $\mathrm{X}$ \\
\hline Tachigalia paniculata Aubl. & AP & & $\mathrm{X}$ & \\
\hline Taralea oppositifolia Aubl. & AP & & $\mathrm{X}$ & \\
\hline Zygia latifolia (L.) Fawc. \& Rendle & $\mathrm{AF}$ & & $\mathrm{X}$ & \\
\hline \multicolumn{5}{|l|}{ 25. FLACOURTIACEAE } \\
\hline Casearia grandiflora Cambess. & $\mathrm{AF}$ & $\mathrm{X}$ & $\mathrm{X}$ & \\
\hline Casearia javitensis Kunth & $\mathrm{AF}$ & & $\mathrm{X}$ & $\mathrm{X}$ \\
\hline \multicolumn{5}{|l|}{ 26. HELICONIACEAE } \\
\hline Heliconia spathocircinata Aristeg. & $\mathrm{HF}$ & & & $\mathrm{X}$ \\
\hline Heliconia acuminata Rich. & $\mathrm{HF}$ & & & $\mathrm{X}$ \\
\hline \multicolumn{5}{|l|}{ 27. HIPPOCRATEACEAE } \\
\hline Cheiloclinium cognatum (Miers) A.C. Sm. & $\mathrm{AP}$ & & $\mathrm{X}$ & \\
\hline Salacia insignis A. C. Sm. & $\mathrm{AF}$ & & & $\mathrm{X}$ \\
\hline
\end{tabular}


TABELA 1: Continuação ...

TABLE 1: Continued ...

\begin{tabular}{|c|c|c|c|c|}
\hline Família/Espécie & $\begin{array}{l}\text { Forma de vida e } \\
\text { grupo funcional }\end{array}$ & Estrato superior & Estrato médio & $\begin{array}{l}\text { Estrato } \\
\text { inferior }\end{array}$ \\
\hline \multicolumn{5}{|l|}{ 28. HYPERICACEAE } \\
\hline Vismia baccifera (L.) Triana \& Planch. & AP & & $\mathrm{X}$ & \\
\hline Vismia guianensis Aubl. Pers. & AP & & & $\mathrm{X}$ \\
\hline \multicolumn{5}{|l|}{ 29. ICACINACEAE } \\
\hline Humirianthera duckei Huber & LF & & $\mathrm{X}$ & $\mathrm{X}$ \\
\hline \multicolumn{5}{|l|}{ 30. LACISTEMATACEAE } \\
\hline Lacistema aggregatum (P.J.Bergius) Rusby & $\mathrm{AF}$ & & $\mathrm{X}$ & \\
\hline \multicolumn{5}{|l|}{ 31. LAMIACEAE } \\
\hline Vitex triflora Vahl & $\mathrm{AF}$ & & $\mathrm{X}$ & \\
\hline \multicolumn{5}{|l|}{ 32. LAURACEAE } \\
\hline Aniba canelilla (Kunth) Mez & $\mathrm{AF}$ & $\mathrm{X}$ & & $\mathrm{X}$ \\
\hline Aniba guianensis Aubl. & $\mathrm{AF}$ & & $\mathrm{X}$ & \\
\hline Aniba taubertiana $\mathrm{Mez}$ & $\mathrm{AF}$ & & & $\mathrm{X}$ \\
\hline Mezilaurus itauba (Meisn.) Taub. ex Mez & $\mathrm{AF}$ & $\mathrm{X}$ & $\mathrm{X}$ & $\mathrm{X}$ \\
\hline Nectandra aff. cissiflora Ness & AP & $\mathrm{X}$ & & \\
\hline Ocotea caudata (Nees) Mez & $\mathrm{AF}$ & & $\mathrm{X}$ & $\mathrm{X}$ \\
\hline Ocotea glomerata (Nees) Mez & $\mathrm{AF}$ & $\mathrm{X}$ & & \\
\hline Ocotea longifolia Kunth & $\mathrm{AF}$ & $\mathrm{X}$ & $\mathrm{X}$ & \\
\hline Ocotea splendens (Meisn.) Baill. & $\mathrm{AF}$ & & $\mathrm{X}$ & \\
\hline \multicolumn{5}{|l|}{ 33. LECYTHIDACEAE } \\
\hline Couratari tenuicarpa A.C. Sm & $\mathrm{AF}$ & $\mathrm{X}$ & & \\
\hline Eschweilera coriacea S. A. Mori & $\mathrm{AF}$ & $\mathrm{X}$ & $\mathrm{X}$ & $\mathrm{X}$ \\
\hline Eschweilera pedicellata (Rich.) S.A. Mori & $\mathrm{AF}$ & $\mathrm{X}$ & $\mathrm{X}$ & \\
\hline Lecythis lurida (Miers) S.A. Mori & $\mathrm{AF}$ & $\mathrm{X}$ & $\mathrm{X}$ & $\mathrm{X}$ \\
\hline Lecythis sp. & $\mathrm{AF}$ & $\mathrm{X}$ & $\mathrm{X}$ & $\mathrm{X}$ \\
\hline \multicolumn{5}{|l|}{ 34. LOGANIACEAE } \\
\hline Strychnos mitscherlichii M.R. Schomb & $\mathrm{AF}$ & & $\mathrm{X}$ & \\
\hline Strychnos parvifolia A. DC. & $\mathrm{AF}$ & & & $\mathrm{X}$ \\
\hline \multicolumn{5}{|l|}{ 35. MALVACEAE } \\
\hline Bombax longipedicellatum Ducke & $\mathrm{AF}$ & $\mathrm{X}$ & & \\
\hline Eriotheca globosa (Aubl.) A. Robyns & $\mathrm{AF}$ & $\mathrm{X}$ & $\mathrm{X}$ & $\mathrm{X}$ \\
\hline Guazuma ulmifolia Lam. & $\mathrm{AF}$ & & & $\mathrm{X}$ \\
\hline Quararibea guianensis Aubl & $\mathrm{AF}$ & $\mathrm{X}$ & $\mathrm{X}$ & $\mathrm{X}$ \\
\hline Quararibea ochroçalyx (K. Schum.) Vischer & $\mathrm{AF}$ & $\mathrm{X}$ & $\mathrm{X}$ & $\mathrm{X}$ \\
\hline Sterculia striata A. St.-Hil. \& Naudin & $\mathrm{AP}$ & & & $\mathrm{X}$ \\
\hline $\begin{array}{l}\text { Theobroma grandiflorum(Willd. ex Spreng.) K. } \\
\text { Schum. }\end{array}$ & AP & $\mathrm{X}$ & & $\mathrm{X}$ \\
\hline Theobroma speciosum Willd. ex Spreng. & $\mathrm{AF}$ & $\mathrm{X}$ & $\mathrm{X}$ & $\mathrm{X}$ \\
\hline Theobroma subincanum Mart. & $\mathrm{AF}$ & $\mathrm{X}$ & $\mathrm{X}$ & \\
\hline \multicolumn{5}{|l|}{ 36. MALPIGHIACEAE } \\
\hline Tetrapterys benthamiana Griseb. & LF & & & $\mathrm{X}$ \\
\hline \multicolumn{5}{|l|}{ 37. MARANTACEAE } \\
\hline Calathea acuminata Steyerm. & HP & & & $\mathrm{X}$ \\
\hline Calathea grandis Petersen & HP & & & $\mathrm{X}$ \\
\hline Calathea lutea Schult. & $\mathrm{HF}$ & & & $X$ \\
\hline Calathea ovata (Nees \& Mart.) Lindl. & $\mathrm{HF}$ & & & $\mathrm{X}$ \\
\hline Ischnosiphon gracilis (Rudge) Körn. & $\mathrm{HF}$ & & & $\mathrm{X}$ \\
\hline Monotagma contractum Huber & $\mathrm{HF}$ & & & $\mathrm{X}$ \\
\hline $\begin{array}{r}\text { Monotagma laxum (Poepp. \& Endl.) Schum. } \\
\text { 38. MELASTOMATACEAE }\end{array}$ & $\mathrm{HF}$ & & & $\mathrm{X}$ \\
\hline Bellucia dichotoma Cogn. & $\mathrm{AP}$ & $\mathrm{X}$ & $\mathrm{X}$ & \\
\hline
\end{tabular}


TABELA 1: Continuação ...

TABLE 1: Continued ...

\begin{tabular}{|c|c|c|c|c|}
\hline Família/Espécie & $\begin{array}{l}\text { Forma de vida e } \\
\text { grupo funcional }\end{array}$ & Estrato superior & Estrato médio & $\begin{array}{l}\text { Estrato } \\
\text { inferior }\end{array}$ \\
\hline $\begin{array}{l}\text { Miconia chrysophylla (Rich.) Urb. } \\
\text { 39. MELIACEAE }\end{array}$ & ArbP & $\mathrm{X}$ & $\mathrm{X}$ & \\
\hline Cedrela odorata $\mathrm{L}$. & $\mathrm{AF}$ & $\mathrm{X}$ & & \\
\hline Guarea guidonia (L.) Sleumer & $\mathrm{AF}$ & $\mathrm{X}$ & $\mathrm{X}$ & $\mathrm{X}$ \\
\hline Guarea kunthiana A. Juss. & $\mathrm{AF}$ & & & $\mathrm{X}$ \\
\hline Guarea purusana C. DC. & $\mathrm{AF}$ & & $\mathrm{X}$ & \\
\hline Guarea quatiana & $\mathrm{AF}$ & & $\mathrm{X}$ & \\
\hline Guarea silvatica C. DC. & $\mathrm{AF}$ & & $\mathrm{X}$ & $\mathrm{X}$ \\
\hline Guarea subsessiliflora C. DC. & $\mathrm{AF}$ & & & $\mathrm{X}$ \\
\hline Trichilia elegans A.Juss. & $\mathrm{AF}$ & & & $\mathrm{X}$ \\
\hline Trichilia micrantha Benth. & $\mathrm{AF}$ & & & $\mathrm{X}$ \\
\hline \multicolumn{5}{|l|}{ 40. MENISPERMACEAE } \\
\hline Abuta grandifolia (Mart.) Sandwith & LF & & $\mathrm{X}$ & $\mathrm{X}$ \\
\hline \multicolumn{5}{|l|}{ 41. METAXYACEAE } \\
\hline Metaxya rostrata (Kunth) C. Presl & $\mathrm{HF}$ & & & $\mathrm{X}$ \\
\hline \multicolumn{5}{|l|}{ 42. MORACEAE } \\
\hline Bagassa guianensis Aubl. & $\mathrm{AF}$ & & $\mathrm{X}$ & $\mathrm{X}$ \\
\hline Brosimum guianensis (Aubl.) Huber & $\mathrm{AF}$ & & $\mathrm{X}$ & $\mathrm{X}$ \\
\hline Brosimum lactescens(S. Moore) C.C. Berg. & $\mathrm{AF}$ & $\mathrm{X}$ & $\mathrm{X}$ & \\
\hline Castilloa ulei Warb. & $\mathrm{AF}$ & $\mathrm{X}$ & $\mathrm{X}$ & $\mathrm{X}$ \\
\hline Clarisia ilicifolia (Spreng.) Lanj. \& Rossberg. & $\mathrm{AF}$ & $\mathrm{X}$ & & $\mathrm{X}$ \\
\hline Clarisia racemosa Ruiz \& Pav. & $\mathrm{AF}$ & $\mathrm{X}$ & & \\
\hline Ficus catappifolia Kunth \& Bouché & $\mathrm{AF}$ & & $\mathrm{X}$ & \\
\hline Maquira guianensis Aubl. & $\mathrm{AF}$ & $\mathrm{X}$ & $\mathrm{X}$ & $\mathrm{X}$ \\
\hline Maquira sclerophylla (Ducke) C.C. Berg & $\mathrm{AF}$ & $\mathrm{X}$ & $\mathrm{X}$ & \\
\hline Naucleopsis caloneura (Huber) Ducke & $\mathrm{AF}$ & & $\mathrm{X}$ & $\mathrm{X}$ \\
\hline Naucleopsis glabra Spruce ex Baill. & $\mathrm{AF}$ & $\mathrm{X}$ & $\mathrm{X}$ & $\mathrm{X}$ \\
\hline \multicolumn{4}{|l|}{ 43. MYRISTICACEAE } & $\mathrm{X}$ \\
\hline Campsoneura ulei Worb. & $\mathrm{AF}$ & & $\mathrm{X}$ & $\mathrm{X}$ \\
\hline Virola michelii Heckel & $\mathrm{AF}$ & $\mathrm{X}$ & $\mathrm{X}$ & $\mathrm{X}$ \\
\hline Virola scabifera & $\mathrm{AF}$ & $\mathrm{X}$ & $\mathrm{X}$ & \\
\hline \multicolumn{5}{|l|}{ 44. MYRTACEAE } \\
\hline Eugenia brachypoda DC. & ArbF & $\mathrm{X}$ & & \\
\hline Eugenia egensis DC. & ArbF & $\mathrm{X}$ & & \\
\hline Eugenia omissa McVaugh & $\mathrm{AF}$ & $\mathrm{X}$ & $\mathrm{X}$ & $\mathrm{X}$ \\
\hline Myrcia bracteata (Rich) DC. & ArbP & & $\mathrm{X}$ & \\
\hline Myrcia deflexa (Poir.) DC. & ArbF & & $\mathrm{X}$ & $\mathrm{X}$ \\
\hline Myrcia paivae O. Berg & ArbP & & & $\mathrm{X}$ \\
\hline \multicolumn{5}{|l|}{ 45. NYCTAGINACEAE } \\
\hline Guapira noxia (Netto) Lundell & AP & & $\mathrm{X}$ & \\
\hline Guapira venosa (Choisy) Lundell & AP & $\mathrm{X}$ & $\mathrm{X}$ & $\mathrm{X}$ \\
\hline $\begin{array}{l}\text { Neea oppositifolia Ruiz \& Pav. } \\
\text { 46. OCHNACEAE }\end{array}$ & ArbF & $\mathrm{X}$ & $\mathrm{X}$ & $\mathrm{X}$ \\
\hline $\begin{array}{l}\text { Ouratea paraensis Huber } \\
\text { 47. OLACACEAE }\end{array}$ & $\mathrm{AF}$ & $X$ & $X$ & \\
\hline Heisteria maguirei Sleumer & $\mathrm{AF}$ & & $\mathrm{X}$ & \\
\hline
\end{tabular}


TABELA 1: Continuação ...

TABLE 1: Continued ...

\begin{tabular}{|c|c|c|c|c|}
\hline Família/Espécie & $\begin{array}{l}\text { Forma de vida e } \\
\text { grupo funcional }\end{array}$ & Estrato superior & Estrato médio & $\begin{array}{l}\text { Estrato } \\
\text { inferior }\end{array}$ \\
\hline $\begin{array}{l}\text { Minquartia guianensis Aubl. } \\
\text { 48. PIPERACEAE }\end{array}$ & $\mathrm{AF}$ & & $\mathrm{X}$ & \\
\hline Peperomia sp. & $\mathrm{HF}$ & & & $\mathrm{X}$ \\
\hline Piper aduncum L. & ArbF & & & $\mathrm{X}$ \\
\hline Piper cf carniconnectivum C.DC & ArbP & & & $\mathrm{X}$ \\
\hline $\begin{array}{l}\text { Piper hispidum Sw. } \\
\text { 49. POACEAE }\end{array}$ & ArbP & & & $\mathrm{X}$ \\
\hline Ichnanthus breviscrobs Döll & $\mathrm{HF}$ & & & $\mathrm{X}$ \\
\hline Merostachys sp. & $\mathrm{HF}$ & & & $\mathrm{X}$ \\
\hline Olyra latifolia L. & $\mathrm{HF}$ & & & $\mathrm{X}$ \\
\hline Pariana lunata Ness & $\mathrm{HF}$ & & & $\mathrm{X}$ \\
\hline $\begin{array}{l}\text { Pharus glaber Kunth } \\
\text { 50. POLYGONACEAE }\end{array}$ & $\mathrm{HF}$ & & & $\mathrm{X}$ \\
\hline $\begin{array}{c}\text { Coccoloba cf. densifrons C. Mart. ex Meisn. } \\
\text { 51. PTERIDACEAE }\end{array}$ & AP & & & $\mathrm{X}$ \\
\hline Adiantum dolosum Kunze & $\mathrm{HF}$ & & & $\mathrm{X}$ \\
\hline Adiantum latifolium Lam. & $\mathrm{HF}$ & & & $\mathrm{X}$ \\
\hline Adiantum tomentosum Klotzsch & $\mathrm{HF}$ & & & $\mathrm{X}$ \\
\hline Adiantum sp. & $\mathrm{HF}$ & & & $\mathrm{X}$ \\
\hline $\begin{array}{l}\text { 52. QUINACEAE } \\
\text { Lacunaria crenata (Tul.) A.C.Sm. } \\
\text { 53. RUBIACEAE }\end{array}$ & $\mathrm{AF}$ & & $\mathrm{X}$ & \\
\hline Alibertia edulis (Rich.) A. Rich. ex DC. & ArbP & $\mathrm{X}$ & & $\mathrm{X}$ \\
\hline Coussarea sp. & ArbP & & & $\mathrm{X}$ \\
\hline Faramea bracteata Benth. & ArbF & & $\mathrm{X}$ & $\mathrm{X}$ \\
\hline Psychotria kappleri (Miq.) Arg. ex Benoist & ArbF & & $\mathrm{X}$ & $\mathrm{X}$ \\
\hline Psychotria racemosa Rich. & ArbF & & & $\mathrm{X}$ \\
\hline $\begin{array}{l}\text { Remijia glomerata Huber } \\
\text { 54. RUTACEAE }\end{array}$ & ArbP & & $\mathrm{X}$ & $\mathrm{X}$ \\
\hline Galipea jasminiflora (A. St. - Hil.) Engl. & ArbP & & $\mathrm{X}$ & \\
\hline Metrodorea flavida $\mathrm{K}$. Krause & $\mathrm{AF}$ & & & $\mathrm{X}$ \\
\hline Zanthoxylum ekmanii (Urb.) Alain & $\mathrm{AF}$ & & $\mathrm{X}$ & $\mathrm{X}$ \\
\hline $\begin{array}{l}\text { Zanthoxylum regnellianum Engl. } \\
\text { 55. SALICACEAE }\end{array}$ & $\mathrm{AF}$ & & $\mathrm{X}$ & $\mathrm{X}$ \\
\hline $\begin{array}{l}\text { Hasseltia floribunda Kunth } \\
\text { 56. SAPINDACEAE }\end{array}$ & AP & $\mathrm{X}$ & & \\
\hline Allophylus floribundus (Poepp.) Radlk. & $\mathrm{HF}$ & & $\mathrm{X}$ & \\
\hline Cupania diphylla Vahl & $\mathrm{AF}$ & $\mathrm{X}$ & $\mathrm{X}$ & $\mathrm{X}$ \\
\hline Cupania scrobiculata Rich. & $\mathrm{AF}$ & $\mathrm{X}$ & $\mathrm{X}$ & \\
\hline Paullinia bracteosa Radlk. & LF & & & $\mathrm{X}$ \\
\hline Paullinia cf. ferruginea Casar. & LF & & $\mathrm{X}$ & \\
\hline Porocystis sp. & $\mathrm{AF}$ & & $\mathrm{X}$ & \\
\hline Pseudima frutescens (Aubl.) Radlk. & $\mathrm{AF}$ & $\mathrm{X}$ & $\mathrm{X}$ & \\
\hline Serjania paucidentata DC. & $\mathrm{LF}$ & & & $\mathrm{X}$ \\
\hline Serjania sp. & $\mathrm{LF}$ & & $\mathrm{X}$ & $\mathrm{X}$ \\
\hline Talisia carinata Radlk. & ArbF & & $\mathrm{X}$ & \\
\hline Talisia longifolia (Benth.) Radlk. & ArbF & $\mathrm{X}$ & $\mathrm{X}$ & \\
\hline Talisia marleneana (Guarim) Acev.-Rodr. & ArbF & $\mathrm{X}$ & $\mathrm{X}$ & $\mathrm{X}$ \\
\hline Talisia mollis Kumth ex Cambess. & ArbF & $\mathrm{X}$ & $\mathrm{X}$ & \\
\hline $\begin{array}{l}\text { Toulicia guianensis Aubl. } \\
\text { 57. SAPOTACEAE }\end{array}$ & $\mathrm{AF}$ & $\mathrm{X}$ & $\mathrm{X}$ & \\
\hline Chrysophyllum lucentifolium Cronquist & $\mathrm{AF}$ & $\mathrm{X}$ & & \\
\hline
\end{tabular}


TABELA 1: Continuação ...

TABLE 1: Continued ...

\begin{tabular}{|c|c|c|c|c|}
\hline Família/Espécie & $\begin{array}{l}\text { Forma de vida e } \\
\text { grupo funcional }\end{array}$ & Estrato superior & Estrato médio & $\begin{array}{l}\text { Estrato } \\
\text { inferior }\end{array}$ \\
\hline Pouteria sp. & $\mathrm{AF}$ & & $\mathrm{X}$ & \\
\hline Pouteria caimito (Ruiz \& Pav.) Radlk. & $\mathrm{AF}$ & & & $\mathrm{X}$ \\
\hline Pouteria gongrijpii Eyma & $\mathrm{AF}$ & $\mathrm{X}$ & $\mathrm{X}$ & $\mathrm{X}$ \\
\hline $\begin{array}{l}\text { Pouteria jariensis } \text { Pires \& T.D. Penn } \\
\text { 58. SIMAROUBACEAE }\end{array}$ & $\mathrm{AF}$ & $\mathrm{X}$ & & \\
\hline Simaba cedron Planch. & $\mathrm{AF}$ & $\mathrm{X}$ & $\mathrm{X}$ & \\
\hline $\begin{array}{l}\text { Simaruba amara Aubl. } \\
\text { 59. SIPARUNACEAE }\end{array}$ & $\mathrm{AF}$ & $\mathrm{X}$ & $\mathrm{X}$ & \\
\hline Siparuna guianensis Aubl. & $\mathrm{AF}$ & & $\mathrm{X}$ & \\
\hline $\begin{array}{l}\text { Siparuna sp. } \\
\text { 60. SMILACACEAE }\end{array}$ & $\mathrm{AF}$ & & & $\mathrm{X}$ \\
\hline $\begin{array}{c}\text { Smilax siphilitica Humb. \& Bonpl. ex Willd. } \\
\text { 61. SOLANACEAE }\end{array}$ & LF & & & $\mathrm{X}$ \\
\hline $\begin{array}{l}\text { Solanum schlechtendalianum Walp. } \\
62 . \text { TECTARIACEAE }\end{array}$ & ArbF & & $\mathrm{X}$ & $\mathrm{X}$ \\
\hline $\begin{array}{l}\text { Tectaria incisa Cav. } \\
\text { 63. STRELITZIACEAE }\end{array}$ & $\mathrm{HF}$ & & & $\mathrm{X}$ \\
\hline $\begin{array}{l}\text { Phenakospermum guianensis Aubl. } \\
64 . \text { TILIACEAE }\end{array}$ & $\mathrm{HF}$ & & & $\mathrm{X}$ \\
\hline Apeiba burchelli Sprague & AP & $\mathrm{X}$ & & \\
\hline Apeiba echinata Gaertn. & $\mathrm{AF}$ & & & $\mathrm{X}$ \\
\hline $\begin{array}{l}\text { Apeiba tibourbou Aubl. } \\
\text { 65. ULMACEAE }\end{array}$ & $\mathrm{AF}$ & & & $\mathrm{X}$ \\
\hline $\begin{array}{l}\text { Ampelocera edentula Kuhlm. } \\
66 . \text { URTICACEAE }\end{array}$ & $\mathrm{AF}$ & & $\mathrm{x}$ & $\mathrm{X}$ \\
\hline Cecropia obtusa Trécul & $\mathrm{AP}$ & & $\mathrm{X}$ & $\mathrm{X}$ \\
\hline Cecropia palmata Willd. & AP & $\mathrm{X}$ & $\mathrm{X}$ & \\
\hline Cecropia sciadophylla Mart. & AP & $\mathrm{X}$ & $\mathrm{X}$ & \\
\hline $\begin{array}{l}\text { Pourouma guianensis Aubl. } \\
67 . \text { VIOLACEAE }\end{array}$ & AP & & & $\mathrm{X}$ \\
\hline Rinorea flavescens (Aubl. ) Kuntze & $\mathrm{AF}$ & & $\mathrm{X}$ & $\mathrm{X}$ \\
\hline Rinorea neglecta Sandwith & $\mathrm{AF}$ & & $\mathrm{X}$ & $\mathrm{x}$ \\
\hline Rinorea pubiflora (Benth.) Sprague \& Sandwith & $\mathrm{AF}$ & & & $\mathrm{X}$ \\
\hline Rinorea riana Kuntze & ArbP & & $\mathrm{X}$ & $\mathrm{X}$ \\
\hline $\begin{array}{l}\text { Rinoreocarpus longifolia } \text { Spreng. } \\
\qquad 68 . \text { ZINGIBERACEAE }\end{array}$ & ArbP & & & $\mathrm{X}$ \\
\hline Renealmia alpina (Rottb.) Maas & HP & & & $\mathrm{X}$ \\
\hline
\end{tabular}

Os valores de equibilidade nos estratos superior $(0,96 \pm 0,01)$ e médio $(0,97 \pm 0,00)$ foram altos. Embora, o estrato inferior seja o mais rico em espécies $(41,82 \pm 2,50)$, pois contém as plantas herbáceas e as plântulas jovens das arbóreas, o estrato médio apresentou a maior diversidade de Shannon $(3,28 \pm 0,09)$ (Tabela 2).

A riqueza de espécies correspondeu a $70 \%$ da riqueza estimada por Jack-nife de primeira ordem $\left(\mathrm{J}^{1}=418,45\right)$ e a $55,8 \%$ da riqueza estimada por Jack-nife de segunda ordem $\left(\mathrm{J}^{2}=524,49\right)$. A porcentagem de espécies raras foi alta; 138 espécies ocorreram em apenas uma área e 42 espécies ocorreram em duas áreas.

A área basal média do estrato superior foi de $\left(3,08 \pm 0,90 \mathrm{~m}^{2} / \mathrm{ha}\right)$ e do estrato médio foi de $0,36 \pm$ $0,14 \mathrm{~m}^{2} / \mathrm{ha}$. A densidade foi maior no estrato médio do que no estrato superior para todas as formas de vida. Em todos os estratos, a forma de vida que apresentou maior densidade foram as árvores, seguida por arbusto, lianas e herbáceas, exceto no estrato inferior, onde as herbáceas foram mais densas, seguida das árvores (Tabela 2). As espécies florestais predominaram em todos os estratos. Houve diferença significante entre as florestais e pioneiras em todas as formas de vida, em todos os estratos. As 
espécies pioneiras também apresentaram o mesmo padrão (Tabela 3 ).

A PCA mostrou heterogeneidade entre os transectos estudados, sendo que os eixos $1 \mathrm{e}$ 2 explicaram $96 \%$ da variância. Três grupos podem ser visualizados (Figura 1). O grupo formado pelos transectos T1, T2 e T3, tiveram 28 espécies indicadores significativas. Com exceção de quatro espécies, Castilloa ulei, Acacia multipinnata, Symphonia globulifera, Mezilaurus itauba, as outras 24 ocorreram exclusivamente nesse grupo. As espécies que apresentaram maiores valores do IndVal neste grupo foram Adiantum sp., Rinorea flavescens, Monstera obliqua, Oenocarpus distichus, Humirianthera duckei (Tabela 4).

O segundo grupo foi formado pelos transectos T6, T7, T11 e T9 com Duguetia flagellaris, Rinorea neglecta (ambas com os maiores valores de IndVal), Inga alba, Trichilia quadrijuga, Naucleopsis glabra e Oenocarpus bacaba como espécies indicadoras significativas. Os transectos T4, T5, T8 e T10 formam o terceiro grupo, com apenas Adiantum tomentosum como indicadora significativa (Tabela 4).

\section{DISCUSSÃO}

No estrato superior a riqueza e diversidade foram inferiores àquelas encontradas em florestas localizadas nas regiões circunvizinhas, como aquelas estudadas por Morelato e Rosa (1991), Ribeiro et al. (1999) e Francez et al. (2007). As florestas do PA-Benfica vêm sofrendo exploração madeireira há muitos anos (HOMMA et al.,

TABELA 2: Média e erro padrão da área Basal ( $\mathrm{m}^{2} /$ ha), Índice de Shannon (H'), Riqueza (S), Equibilidade (E), Densidade (indivíduos $/ \mathrm{m}^{2}$ ) das 11 áreas estudadas no PA-Benfica, Itupiranga, Pará.

TABLE 2: $\quad$ Mean and standard error of the basal area $\left(\mathrm{m}^{2} / \mathrm{ha}\right)$, Shannon index $\left(\mathrm{H}^{\prime}\right)$, richness (S), Evenness (E), density (individuals $/ \mathrm{m}^{2}$ ) of the 11 areas studied in PA-Benfica, Itupiranga, Pará state.

\begin{tabular}{lccc}
\hline \multirow{2}{*}{ Parâmetros fitossociológicos } & \multicolumn{3}{c}{ Estratos } \\
\cline { 2 - 4 } & Superior & Médio & Inferior \\
\hline Área Basal $\left(\mathrm{m}^{2} / \mathrm{ha}\right)$ & $3,08 \pm 0,90$ & $0,36 \pm 0,14$ & - \\
Shannon $\left(\mathrm{H}^{\prime}\right)$ & $2,70 \pm 0,07$ & $3,27 \pm 0,09$ & $2,50 \pm 0,14$ \\
Riqueza $(\mathrm{S})$ & $17,09 \pm 1,00$ & $30,45 \pm 2,20$ & $41,55 \pm 2,42$ \\
Equibilidade $(\mathrm{E})$ & $0,96 \pm 0,01$ & $0,97 \pm 0,00$ & $0,67 \pm 0,03$ \\
Densidade (ind./ $\left.\mathrm{m}^{2}\right)$ & - & - & - \\
$\quad$ - Árvores & $3,88 \pm 0,14$ & $10,08 \pm 0,76$ & $4,02 \pm 0,55$ \\
$\quad$ - Arbustos & $0,63 \pm 0,10$ & $1,69 \pm 0,23$ & $0,53 \pm 0,08$ \\
• Lianas & 0 & $0,43 \pm 0,23$ & $0,39 \pm 0,08$ \\
$\quad$ - Herbáceas & 0 & $0,25 \pm 0,08$ & $4,15 \pm 1,05$ \\
\hline
\end{tabular}

TABELA 3: Média e erro padrão do número de espécies pioneiras (P) e florestais (F), por forma de vida, coletadas nos três estratos nos 11 fragmentos florestais estudados no PA-Benfica, município de Itupiranga, Pará.

TABLE 3: Mean and standard error of the number of pioneer species (P) and forest (F), by life form, of the 11 areas studied in PA-Benfica, Itupiranga, Pará state.

\begin{tabular}{|c|c|c|c|c|c|}
\hline & Árvores & Arbusto & Lianas & Palmeiras & Herbáceas \\
\hline \multicolumn{6}{|c|}{ Estrato superior } \\
\hline$\overline{\text { Espécies -P }}$ & $1,82 \pm 0,33 \mathrm{a}$ & $0,18 \pm 0,12 \mathrm{a}$ & 0 & $0 \mathrm{a}$ & 0 \\
\hline Espécies - F & $12,36 \pm 0,91 \mathrm{~b}$ & $2,18 \pm 0,35 \mathrm{~b}$ & 0 & $0,64 \pm 0,20 \mathrm{~b}$ & 0 \\
\hline \multicolumn{6}{|c|}{ Estrato médio } \\
\hline Espécies -P & $2,82 \pm 0,54 \mathrm{a}$ & $1,00 \pm 0,27 \mathrm{a}$ & $0,09 \pm 0,09 \mathrm{a}$ & $0 \mathrm{a}$ & $0,09 \pm 0,0 \mathrm{a}$ \\
\hline Espécies - F & $20,45 \pm 1,61 b$ & $3,36 \pm 0,39 b$ & $1,18 \pm 0,62 \mathrm{a}$ & $0,91 \pm 0,28 \mathrm{~b}$ & $0,45 \pm 0,16 \mathrm{a}$ \\
\hline \multicolumn{6}{|c|}{ Estrato inferior } \\
\hline Espécies -P & $2,09 \pm 0,39 \mathrm{a}$ & $0,91 \pm 0,25 \mathrm{a}$ & $0 \mathrm{a}$ & $0 \mathrm{a}$ & $0,82 \pm 0,26 \mathrm{a}$ \\
\hline Espécies - F & $18,36 \pm 1,44 \mathrm{~b}$ & $3,91 \pm 0,56 b$ & $4,82 \pm 0,30 \mathrm{~b}$ & $2,91 \pm 0,25 \mathrm{~b}$ & $7,73 \pm 0,49 b$ \\
\hline
\end{tabular}

Em cada estrato, os números seguidos da mesma letra na vertical não apresentam diferença significativa (Anova de um critério, teste de Bonferroni, $\mathrm{p}<0,001)$. 


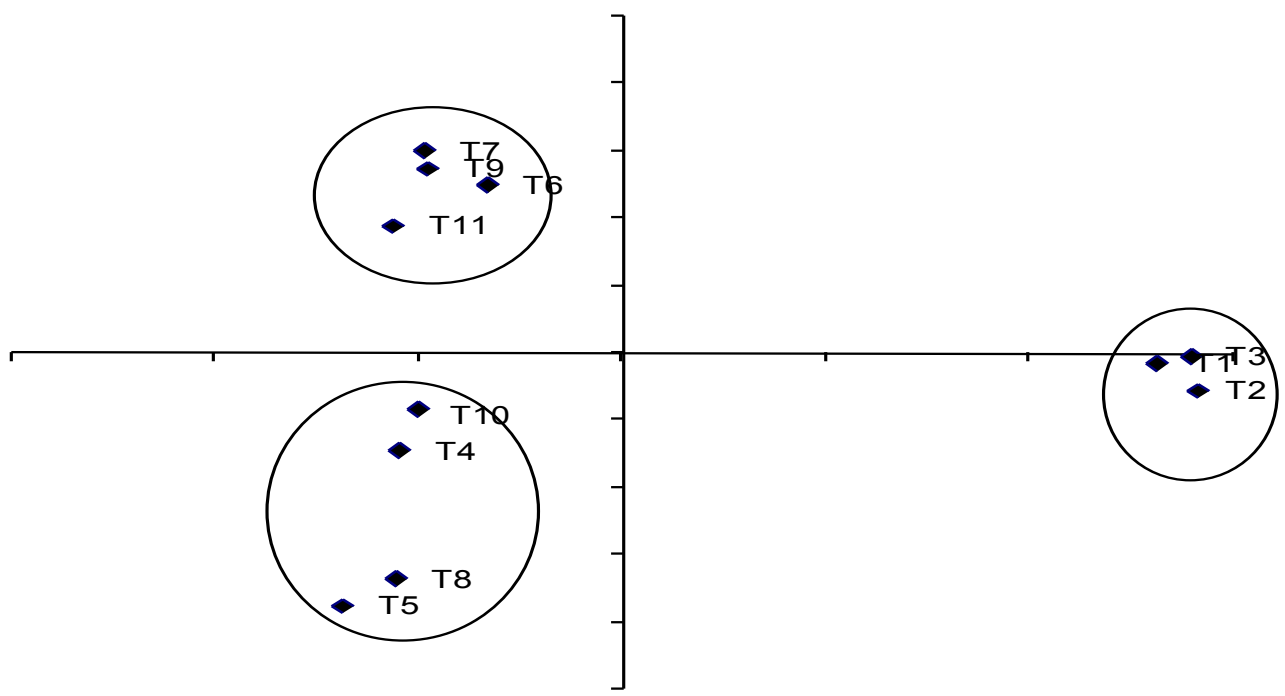

FIGURA 1: Resultado da análise de componente principal das 11 áreas baseado na abundância das 110 espécies encontradas no PA-Benfica, Itupiranga, Pará.

FIGURE 1: PCA Ordination of 11 areas based on abundance of 110 species in PA-Benfica, Itupiranga, Pará state.

2000), todavia ainda apresentam espécies de valor comercial encontradas em florestas secundárias do Estado do Pará, como Lecythis lurida, Eschweilera coriacea, Inga alba, Jacaranda copaia (AMARAL et al., 2000; ALVINO et al., 2005).

A ocorrência de espécies típicas do subbosque, tais como Duguetia flagellaris, Rinorea flavescens e Rinorea neglecta, juntamente com as jovens das espécies arbóreas que pertencem ao dossel, tais como Protium apiculatum, Poecilanthe effusa, contribuiu para que o estrato médio apresentasse o maior índice de diversidade de Shannon. Embora esse resultado fosse esperado para o estrato inferior que apresenta todas as formas de vida, ele indica um bom desenvolvimento das espécies lenhosas. Uma boa regeneração das espécies lenhosas também foi indicada pela grande riqueza de plântulas das árvores no estrato inferior.

Um quinto das espécies lenhosas encontravase exclusivamente no estrato inferior. Esse resultado pode ser devido à dificuldade de visualização de algumas lianas nos estratos superiores, como Dioclea malococarpa, Doliocarpus dentatus, Machaerium ferox, Memora contracta, Memora magnifica, Memora cf. flaviflora Pachyptera kerere, Paullinia bracteosa, Pleonotoma jasminifolia, Pleonotoma cf dendrotricha, Serjania paucidentata, Smilax sylphilica e Tetrapterys benthamiana ou devido à presença de espécies arbóreas que permanecem longo tempo nos estágios iniciais do desenvolvimento, aguardando melhores condições ambientais para o desenvolvimento, como Aniba taubertiana, Apeiba echinata, Apeiba tibourbou, Attalea maripa, Guarea kunthiana, Guarea subsessiliflora, etc.

No estrato inferior, a riqueza das espécies herbáceas (35 espécies em 0,055 ha) pode ser considerada alta, se comparada com outros estudos que consideram essa forma de vida. Silva (2003) encontrou 28 espécies em 0,22 ha em uma floresta tropical estacional submontana, no Estado de Roraima e Costa et al. (2005) encontraram 87 espécies em 2,95 ha da Reserva Ducke, Estado do Amazonas. Considerando as diferenças metodológicas, a riqueza de espécies herbáceas no estrato inferior encontrada no PA-Benfica é bom indicativo das boas condições ambientais nesse microssítio, pois espécies herbáceas, incluindo as samambaias, possuem adaptações estruturais e fisiológicas associadas a esses ambientes e são boas indicadoras de mudanças ambientais, por serem mais sensíveis às modificações ambientais (POULSEN e BALSLEV, 1991; ZUQUIM et al., 2008).

Os valores de equibilidade apresentados pelos estratos mostram alta equibilidade em cada um deles. $\mathrm{O}$ estrato inferior apresentou uma menor equibilidade provavelmente devido ao fato de agrupar nesse estrato diferentes formas de vida e diferentes estágios de vida, como as plântulas e jovens de espécies lenhosas. 
TABELA 4: Espécies indicadoras nos três grupos florísticos de fragmentos florestais, do PA-Benfica. Soma das densidades, Valor do IndVal, Frequência no grupo e forma de vida e grupo funcional. $\mathrm{F}=$ florestal, $\mathrm{P}=$ pioneira, $\mathrm{L}=$ lenhosa, $\mathrm{H}$ = herbácea.

TABLE 4: Indicators species in the three floristic groups of forest fragments. Sum of densities, IndVal value, frequency in group, and life form and functional group. $\mathrm{F}=$ forest, $\mathrm{P}=$ pioneer, $\mathrm{L}=$ woody, $\mathrm{H}=$ herb.

\begin{tabular}{|c|c|c|c|c|c|}
\hline Grupo & Espécies & $\begin{array}{l}\text { Densidade } \\
\text { no grupo } \\
\text { (ind. } / \mathrm{m}^{2} \text { ) }\end{array}$ & IndVal & $\begin{array}{c}\text { Frequência } \\
\text { no grupo }\end{array}$ & $\begin{array}{l}\text { Forma de } \\
\text { vida e grupo } \\
\text { funcional }\end{array}$ \\
\hline 1 & Adiantum sp. & 13,94 & 100 & 3 & $\mathrm{HF}$ \\
\hline 1 & Rinorea flavescens (Aubl.) Kuntze & 5,07 & 100 & 3 & LF \\
\hline 1 & Crepidospermum goudotianum (Tul.) Triana \& Planch. & 2,60 & 89,89 & 3 & LF \\
\hline 1 & Monstera obliqua Miq. & 2,24 & 100 & 3 & $\mathrm{HF}$ \\
\hline 1 & Castilloa ulei Warb. & 2,16 & 81,93 & 6 & LF \\
\hline 1 & Acacia multipinnata Ducke & 1,60 & 88,89 & 4 & $\mathrm{HF}$ \\
\hline 1 & Merostachys sp. & 1,60 & 100 & 3 & $\mathrm{HF}$ \\
\hline 1 & Oenocarpus distichus Mart. & 1,60 & 100 & 3 & LF \\
\hline 1 & Siparuna krukovii A. C. Smith & 1,60 & 100 & 3 & LF \\
\hline 1 & Naucleopsis caloneura (Huber) Ducke & 1,52 & 100 & 3 & LF \\
\hline 1 & Symphonia globulifera L.f. & 1,28 & 85,49 & 5 & LF \\
\hline 1 & Humirianthera duckei Huber & 1,24 & 100 & 3 & LF \\
\hline 1 & Machaerium madeirense Pittier & 1,20 & 100 & 3 & LF \\
\hline 1 & Rinorea pubiflora (Benth.) Sprague \& Sandwith & 1,18 & 100 & 3 & LF \\
\hline 1 & Pariana lunata Nees & 1,17 & 100 & 3 & $\mathrm{HF}$ \\
\hline 1 & Couepia sp. & 1,17 & 100 & 3 & LF \\
\hline 1 & Pourouma guianensis Aubl. & 1,16 & 100 & 3 & LP \\
\hline 1 & Vismia guianensis Aubl. Pers. & 1,16 & 100 & 3 & LP \\
\hline 1 & Mezilaurus itauba (Meisn.) Taub ex. Mez. & 0,92 & 90,57 & 4 & LF \\
\hline 1 & Lecythis sp. & 0,86 & 100 & 3 & LF \\
\hline 1 & Serjania sp. & 0,84 & 74,75 & 3 & LF \\
\hline 1 & Quararibea ochroçalyx (K. Schum.) Vischer & 0,78 & 100 & 3 & LF \\
\hline 1 & Annona montana Macfad. & 0,72 & 100 & 3 & LP \\
\hline 1 & Guarea guidonia (L.) Sleumer & 0,71 & 100 & 3 & LF \\
\hline 1 & Carpotroche sp. & 0,70 & 100 & 3 & LF \\
\hline 1 & Myrcia deflexa (Poir.) DC. & 0,65 & 100 & 3 & LF \\
\hline 1 & Calathea ovata (Nees \& Mart.) Lindl. & 0,62 & 100 & 3 & $\mathrm{HF}$ \\
\hline 1 & Casearia grandiflora A. St.-Hil. & 0,52 & 100 & 3 & LF \\
\hline 2 & Duguetia flagellaris Huber & 10,32 & 100 & 8 & LF \\
\hline 2 & Rinorea neglecta Sandwith & 5,92 & 100 & 8 & LF \\
\hline 2 & Naucleopsis glabra Spruce ex Baill. & 2,88 & 87,5 & 7 & LF \\
\hline 2 & Inga alba Willd. & 2,70 & 100 & 8 & LF \\
\hline 2 & Trichilia quadrijuga Kunth & 1,80 & 88.89 & 5 & LP \\
\hline 2 & Oenocarpus bacaba Mart. & 1,72 & 81.4 & 6 & LF \\
\hline 3 & Adiantum tomentosum Klotzsch & 5,02 & 85.26 & 6 & $\mathrm{HF}$ \\
\hline
\end{tabular}

Os três grupos apresentados na análise dos componentes principais parecem estar relacionados aos diferentes níveis de perturbações. No primeiro grupo (formados pelos transectos T1, T2 e T3) embora a maioria das espécies indicadoras sejam classificadas como florestais, a presença de espécies indicadoras pioneiras como Vismia guianensis, Pouroma guinanensis e Annona montana testemunham uma recente perturbação. Através da fisionomia, foi possível observar vestígios de forte perturbação e até vestígios de queimadas que ocorreram nos fragmentos desses transectos. O eixo 2 da PCA foi responsável por separar os transectos queimados daqueles não queimados.

Os outros dois grupos, separados pelo eixo 1 , sofreram menos impacto que o primeiro grupo, mas 
a dominância de espécies florestais do sub-bosque, como Duguetia flagellaris, Rinorea neglecta (fortes indicadoras do segundo grupo) e de espécie do chão florestal, como Adiantum tomentosum (indicadora do terceiro grupo) revelam diferenças estruturais dos grupos, sendo que o segundo grupo pode estar apresentando dominância do sub-bosque devido a danos causados no estrato superior e inferior e o terceiro grupo com um ambiente mais estável, com samambaias do chão florestal predominando.

$\mathrm{O}$ gênero Adiantum é comum em subbosque de floresta tropical (COSTA, 2004) e Rinorea flavescens também é uma espécie amplamente encontrada no sub-bosque das florestas neotropicais, assim como Duguetia flagellaris (OLIVEIRA e AMARAL, 2005; FRANCEZ et al., 2007).

A alta porcentagem de espécies florestais encontrada em todos os grupos formados é um bom indicativo do estado de conservação das florestas. Como essas florestas são utilizadas para a subsistência dos agricultores (caça, coleta de frutos, sementes e madeira), era esperada uma maior porcentagem de espécies pioneiras devido a essas perturbações.

No PA-Benfica pode-se dizer que o processo de pecuarização, a exploração madeireira e a agricultura familiar vêm mantendo uma boa matriz florística, uma vez que o número de espécies de ambientes florestais foi significativo e que a maioria das espécies lenhosas se encontrava nos três estratos estudados, revelando bom potencial regenerativo. Além disso, o grande número de espécies herbáceas encontrado no estrato inferior são espécies típicas do chão das florestas tropicais, fato que pode indicar que os níveis de perturbação ainda não foram significativos para que houvesse mudança florística nesse componente da floresta.

No entanto, como mostrado por Sampaio (2008), a paisagem do PA-Benfica vem sofrendo forte diminuição dos remanescentes florestais nos últimos anos. O problema maior está na manutenção dos fragmentos a médio e longo prazo e não na qualidade dos remanescentes existentes, embora a qualidade possa mudar a médio e longo prazo, necessitando, para contornar essa situação, de estudos de monitoramento. Segundo Ferreira et al. (2005) para se pensar em conservação é preciso romper com o modelo de ocupação tradicional da Amazônia e adotar soluções para subsidiar as decisões de planejamento socioambiental e desenvolvimento econômico em bases sustentáveis. Respeitar as áreas de Reserva Legal e Áreas de
Preservação Permanente seria um bom começo.

\section{AGRADECIMENTOS}

Este trabalho foi financiado pelos projetos: (1) "Biodiversidade e Funcionamento do Solo no Contexto da Agricultura Familiar na Amazônia" (CNPq/IRD/UFRA Processo No 91056/00-0); e, (2) "Manejo e Biodiversidade dos Recursos Naturais na Amazônia" (IFB/IRD/UFRA). Os autores agradecem às pessoas de Benfica por permitirem o trabalho em suas propriedades; Sr. Deurival da Costa Carvalho pela ajuda no campo; Sr. Manoel Cordeiro pela identificação das plantas; Msc. Carlos Henrique Franciscon pela ajuda na revisão dos nomes científicos e sistema de classificação.

\section{REFERÊNCIAS BIBLIOGRÁFICAS}

ALVINO, F. O.; SILVA, M. F. F.; RAYOL, B. P. Potencial de uso das espécies arbóreas de uma floresta secundaria na zona Bragantina, Pará, Brasil. Acta Amazonica, v. 35, n. 4, p. 413-420, 2005.

AMARAL, I. L.; MATOS, F. D. A.; LIMA, J. Composição florística e estrutural de um hectare de floresta densa de terra firme no Rio Uatumã, Amazônia, Brasil. Acta Amazonica, v. 30, p. 377 392.

ANGIOSPERM PHYLOGENY GROUP II. An update of the Angiosperm Phylogeny Group classification for the orders and families of flowering plants: APG II. Botanical Journal of the Linnaean Society, v. 141, n. 4, p. 399-436, 2003. BENGTSSON, J. et al. Reserves, resilience and dynamics landscapes. Ambio, v. 32, p. 389-396, 2003.

BIERREGAARD JR. R. O. et al. Principles of forest fragmentation and conservation in the Amazon. In: BIERREGAARD, R.O. et al. (Eds.) Lessons from Amazonia: The Ecology and Conservation of a Fragmented Forest. New Haven, Connecticut: Yale University Press, 2001. p. 371-385.

BRANDON, K. et al. Brazilian Conservation: Challenges and Opportunities. Conservation Biology, v. 19, n. 3, p. 595-600, 2005.

COSTA, F. R. C. Structure and composition of the ground-herb community in a terra-firme Central Amazonian forest. Acta Amazonica, v. 34, n. 1, p. 53-59, 2004.

COSTA, F. R. C.; MAGNUSSON, W. E; LUIZÃO, R. C. Mesoscale distribution patterns of Amazonian understoryhebs in relation to topography, soil and 
watersheds. Journal of Ecology, v. 93, p. 863-878, 2005.

CULLEN JR, L..; ALGER, K.; RAMBALDI, D. M. Land reform and biodiversity conservation in Brazil in the 1990: conflict and the articulation of mutual interests. Conservation Biology, v. 19, p. 747-755, 2005.

DAUBER, J. et al. Landscape structure as an indicator of biodiversity: matrix effects on species richness. Agriculture, Ecosystems and Environment, v. 98, p. 321-329, 2003.

DUFRÊNE, M.; LEGENDRE, P. Species assemblages and indicator species the need for flexible asymmetrical approach. Ecological Monographs, v. 63, n. 3, p. 345- 366, 1997.

DUNCAN, R. S. Tree recruitment from on-site versus off-site propagule sources during tropical forest succession. New Forests, v. 31, p. 131-150, 2006.

FEARNSIDE, P. M. Desmatamento na Amazônia: dinâmica, impactos e controle. Acta Amazonica, v. 36, n. 3, p. 395-400, 2006.

FERREIRA, L. V.; VENTICINQUE, E.; ALMEIDA, S. S. O desmatamento na Amazônia e a importância das áreas protegidas. Estudos Avançados, v. 19, p. 157-166, 2005.

FOX, J. et al. Shifting cultivation: a new paradigm for managing tropical forests. Bioscience, v. 50, p. 521-528, 2000.

FRANCEZ, L. M. B.; CARVALHO, J. O. P.; JARDIM, F. C. S. Mudanças ocorridas na composição florística em decorrência da exploração florestal em uma área de floresta de terra firme na região de Paragominas, PA. Acta Amazonica, v. 37, n. 2, p. 219-228, 2007.

HARVEY, C. A. et al. Integrating Agricultural Landscapes with Biodiversity Conservation in the Mesoamerican Hotspot. Conservation Biology, v. 22, p. 8-15, 2007.

HOMMA, A. K. O. et al. A destruição dos recursos naturais: o caso da castanha-do-pará no sudeste paraense. Belém: Embrapa Amazônia Oriental, 2000. $74 \mathrm{p}$.

KÖHLER, P. et al. Simulating the Long-term Response of Tropical Wet Forests to Fragmentation. Ecosystems, v. 6, p. 114-128, 2003.

KRAMER, K. U.; GREEN, P. S. Pteridophytes and Gymnosperms. In: KUBITZKI, K. The Families and Genera of Vascular Plants. New York: Springer-Verlag, 1990. p. 1-404.

LAURANCE, W. F.; GASCON, C. How to creatively fragment a landscape. Conservation Biology, v. 11, n. 2, p. 577-279, 1997.

MAGURRAN, A. E. Ecological Diversity and its Measurement. London: Cambridge University Press, 1998. 179 p.

MCCUNE, B. Improving community analysis with the Beals smoothing function. Ecoscience, v. 1, p. 82-86, 1994.

MITJA, D. et al. Plant species richness and floristic composition change along a rice-pasture sequence in substence farms of Brazilian Amazon (Benfica, State of Pará). Agriculure, Ecossystems and Enviroment, v. 124, p. 72-84, 2008.

MITTERMEIER, R. A. et al. A Brief History of Biodiversity Conservation in Brazil. Conservation Biology, v. 19, n. 3, p. 601-607, 2005.

MORELATTO, L. P.; ROSA, N. A. Caracterização de alguns tipos de vegetação na região amazônica, Serra dos Carajás, Pará, Brasil. Revista Brasileira de Botânica, v. 14, p. 1-14, 1991.

MUCHAGATA, M.; BROWN, K. Cows, colonists and trees: rethinking cattle and environmental degradation in Brazilian Amazonia. Agricultural Systems, v. 6, p. 797-816, 2003.

NEPSTAD, D. C. et al. A comparative study of tree establishment in abandoned pasture and mature forest of eastern Amazonia. Oikos, v. 76, p. 25-39, 1996.

OLIVEIRA, A. N.; AMARAL, I. L. Aspectos florísticos, fitossociologicos e ecológicos de um sub-bosque de terra firme na Amazônia Central, Amazonas, Brasil. Acta Amazonica, v. 35, n. 1, p. 1-16, 2005.

PALMER, M. W. The estimating species richness the second-order Jacknife reconcidered. Ecology, v. 72, p. 1512-1513, 1991.

PALMER, M. W. The estimation of species richness by extrapolation. Ecology, v. 71, p. 1195-1198, 1990. PASQUIS, R. et al. Réforme agraire en Amazonie: bilan et perspectives. Cahiers Agriculture, v. 14, n. 1, p. 35-39, 2005.

PIMENTEL, D. et al. Conserving biological diversity in agricultural/forestry systems. BioScience, v. 42, p. 354-362, 1992.

POULSEN, A. D.; BALSLEV, H. Abundance and cover of ground herbs in Amazonian rainforest. Journal of Vegetation Science, v. 2, p. 315-322, 1991.

RADAMBRASIL. RADAM, folha SB.22 Araguaia e parte da folha SC.22 Tocantins, v. 4. Rio de Janeiro, Brasil: Departamento Nacional de Produção Mineral, 1974.

REYNAL, V. et al. Agriculturas familiares e 
desenvolvimento em frente pioneira amazônica. Edição bilíngüe: Português/Francês. Brasília, Brasil: LASAT/CAT/GRET/UAG, 1995.

RIBEIRO, R. J. et al. Estudo fitossociológico nas regiões de Carajás e Marabá, Pará, Brasil. Acta Amazonica, v. 29, n. 2, p. 207-222, 1999.

SAMPAIO, S. M. N. Dinâmica da paisagem e complexidade espacial de um Projeto de Assentamento da Amazônia Oriental. 2008. 175 f. Tese (Doutorado em Ciências Agrárias) Universidade Federal Rural da Amazônia, Belém, 2008.

SILVA, U. S. C. Fitossociologia do componente arbóreo e não arbóreo de uma floresta tropical em Cantá - RR. 2003. 63 f. Dissertação (Mestrado em Ciências Florestais) - Universidade Federal Rural da Amazônia, Belém, 2003.

SOARES-FILHO, B. S. et al. Simulating the response of land-cover changes to road paving and governance along a major Amazon highway: The Santarém-Cuiabá corridor. Global Change Biology, v. 10, n. 5, p. 745-764, 2004.

SOMBROEK, W. Amazon landforms and soils in relation to biological diversity. Acta Amazonica, v. 30, p. 81-100, 2000.

TABARELLI, M.; GASCON, C. Lessons fron fragmenetation research: improviment management and policy guidelines for biodiversity conservation. Conservation Biology, v. 19, p. 734-739, 2005.

TREWAVAS, A. J. The population/ biodiversity paradox. Agricultural efficiency to save wilderness. Plant Physiology, v. 125, n. 174-179, 2001.

VANDERMEER, J. H.; CARVAJAL, R. Metapopulation dynamics and the quality of the matrix. The American Naturalist, v. 158, p. 211220, 2001.

VANDERMEER, J. H.; PERFECTO, I. The Agricultural Matrix and a Future Paradigm for Conservation. Conservation Biology, v. 21, n. 1, p. 274-277, 2007.

VANDERMEER, J. H.; PERFECTO, I. The future of farming and conservation. Science, v. 308, p. 1257-1258, 2005.

VIEIRA I. C. G; PROCTOR, J. Mechanisms of plant regeneration during succession after shifting cultivation in eastern Amazonian. Plant Ecology, v. 192, p. 303-315, 2007.

ZAR, J. H. Biostatistical Analysis. 3rd ed. New Jersey, EUA: Prentice Hall International, 1996. $718 \mathrm{p}$.

ZUQUIM, G. etal. Guide to the ferns and lycophytes of REBIO Uatumã - Central Amazonia. Manaus: INPA, 2008. 316 p. 